\title{
Andrew's Bridge: Achieving Esthetics with a Prosthetic Alternative-Managing Severe Anterior Ridge Defects in Operated Case of Cleft Lip and Palate
}

\author{
${ }^{1}$ Neha Hajira, ${ }^{2}$ Pulkit Khandelwal, ${ }^{3} \mathrm{HS}$ Shashidhar, ${ }^{4}$ Harleen Sachdeva, ${ }^{5}$ Sumit Khare
}

\begin{abstract}
Reconstruction of large anterior ridge defects is often a prosthodontic challenge. Such defects require closure of the defect along with the replacement of the missing teeth so as to achieve proper speech and esthetics. Classifying these defects in the preoperative examination helps to diagnose and predict the prognosis and technical difficulties to be encountered. The aim and purpose of this case report is to describe the management of one such case with missing anterior teeth and severe anterior ridge defects. We also describe the process of fabrication of Andrew's bridge (a fixed-removable partial denture) to treat this Siebert's class III anterior ridge defect.
\end{abstract}

Keywords: Andrew's bridge, Cleft, Fixed-removable partial denture, Prosthesis, Ridge defect.

How to cite this article: Hajira N, Khandelwal P, Shashidhar HS, Sachdeva H, Khare S. Andrew's Bridge: Achieving Esthetics with a Prosthetic Alternative-Managing Severe Anterior Ridge Defects in Operated Case of Cleft Lip and Palate. Int J Prosthodont Restor Dent 2016;6(4):93-97.

Source of support: Nil

Conflict of interest: None

\section{INTRODUCTION}

Congenital or acquired defects in the oral and maxillofacial region are functionally mutilating and esthetically unacceptable. These defects should be reconstructed or rehabilitated immediately. The goals of such reconstruction

\footnotetext{
${ }^{1,2}$ Senior Lecturer, ${ }^{3}$ Professor, ${ }^{4,5}$ Reader

${ }^{1}$ Department of Prosthodontics, Darshan Dental College \& Hospital, Udaipur, Rajasthan, India

${ }^{2}$ Department of Oral and Maxillofacial Surgery, Pacific Dental College \& Hospital, Udaipur, Rajasthan, India

${ }^{3}$ Department of Prosthodontics, College of Dental Sciences Davangere, Karnataka, India

${ }^{4}$ Department of Prosthodontics, RKDF Dental College and Research Centre, Bhopal, Madhya Pradesh, India

${ }^{5}$ Department of Prosthodontics, People's Dental Academy Bhopal, Madhya Pradesh, India

Corresponding Author: Pulkit Khandelwal, Senior Lecturer Department of Oral and Maxillofacial Surgery, Pacific Dental College \& Hospital, Udaipur, Rajasthan, India, Phone: +91-9049831636, e-mail: khandelwal.pulkit22@gmail.com
}

and rehabilitation include restoration of form, function, and esthetics. Complete esthetic surgical replacement of the defective oral tissues is difficult and unpredictable, particularly when a greater degree of the deficient residual ridge is present due to congenital anomaly like cleft alveolus and palate. ${ }^{1}$

Treatment options in such cases included implantsupported fixed dental prosthesis (FDP) following autogenous bone grafting, conventional FDP, a fixed-removable partial denture (RPD), and a conventional RPD. Andrew's bridge system is a fixed-removable prosthesis indicated in patients with large ridge defects. Dr James Andrews first introduced this fixed-removable prosthesis. It consists of a fixed retainer and removable pontics. This fixed-RPD has a pontic assembly that can be removed by the patient. The retainers are either porcelain fused to metal or full veneer metal, which are permanently cemented to the abutments. The retainers are joined with prefabricated castable bars and then cast together, or a prefabricated metal bar is soldered to the metal copings after casting. The removable pontics are retained by a clip on the intaglio surface, which fits precisely over the bar attachment. ${ }^{1}$

Primary indication for this restoration are cases where the abutments are capable for supporting an FDP, but the residual ridge has been severely deficient because of congenital defects, trauma, or any other pathology, so that a conventional FDP would not adequately restore patient's missing teeth and supporting structures. Fixed-RPDs are also indicated for patients with substantial supportive tissue loss and when the relationship of the arches and/or esthetic positioning of the prosthetic teeth create problems for placement of a conventional FPD. ${ }^{1}$

This case report describes the fabrication of a fixedRPD using the Andrew's bridge concept. Removable prosthesis was retained by ball attachments supported by a bar attached to fixed retainers on either side of the edentulous space. This prosthesis was fabricated to achieve the goals of esthetics, phonetics, comfort, hygiene, and favorable stress distribution to the abutments and soft tissues.

\section{CASE REPORT}

A 27-year-old male patient reported with chief complaint of missing upper and lower front teeth and unesthetic 
appearance while smiling. He had unilateral cleft lip and palate on left side, which was surgically corrected when patient was around 1 to 2 years of age. On extraoral examination, facial asymmetry was evident. Scar of previous surgery was present extending from columella of nose to vermillion border of upper lip extending through the upper lip (Fig. 1). Intraorally, maxillary left central and lateral incisors and mandibular both central incisors $(21,22,31,41)$ were congenitally missing. Maxillary right central incisor (11) was mesially rotated, and mandibular both lateral incisors $(32,42)$ were mobile (grade II mobility) with marked gingival recession. Crossbite was present in left canine region. There was marked ridge defect seen on anterior maxillary ridge in left incisal region and anterior mandibular region. The defect in maxillary arch was measured to be approximately $1 \mathrm{~cm}$ from the gingival margin of left canine to the deepest point on the defect. In mandibular arch also, the defect was measured to be approximately $1 \mathrm{~cm}$ from cementoenamel junction of lateral incisors (Fig. 2). Orthopantomogram revealed marked bone loss over the defect areas (Fig. 3).

The defect was classified to be Seibert's class III (combined horizontal and vertical bone loss). On measuring

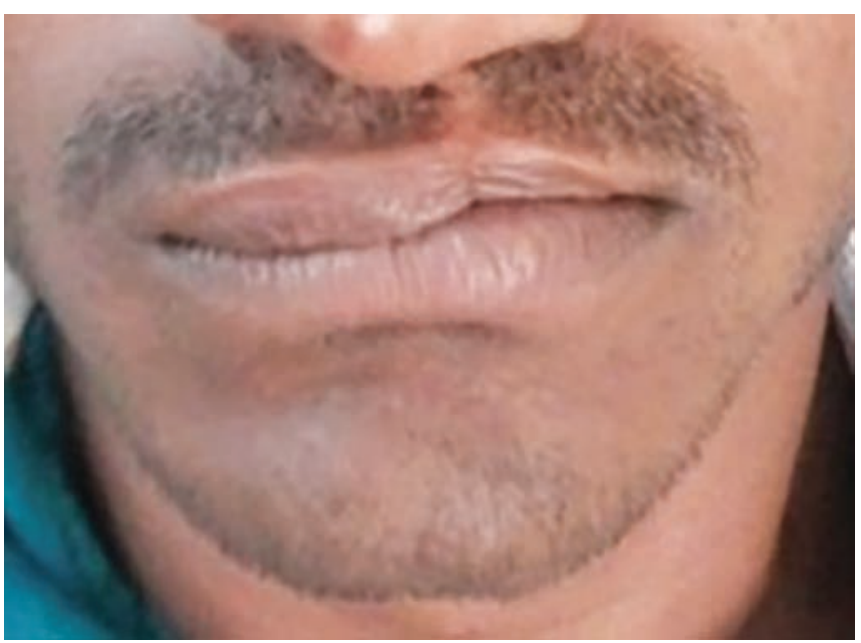

Fig. 1: Extraoral view

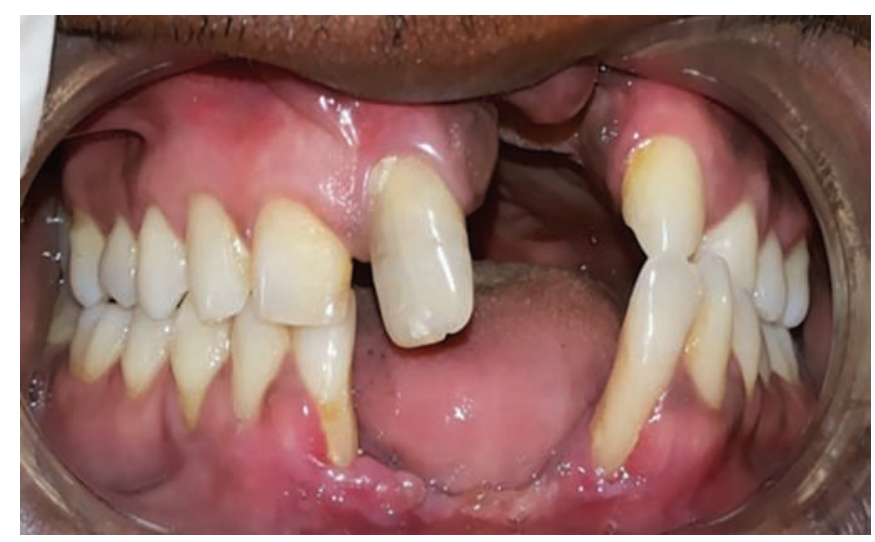

Fig. 2: Preoperative view the defect, it was found to have a severe vertical defect $>6 \mathrm{~mm}(10 \mathrm{~mm})$ and severe horizontal defect $>6 \mathrm{~mm}$ $(8 \mathrm{~mm})$. Treatment options in our case also included implant-supported FDP following autogenous bone graft procedure, conventional FDP, a fixed-RPD, and a conventional RPD. Implant placement would have required bone grafting surgeries. However, patient strongly refused to undergo any surgical reconstructive procedures. The FPD can restore function and esthetics only to a limited extent and the defect would have again required corrections by soft tissue grafts and bone grafts to render it suitable for FPD. The conventional removable prosthesis could not satisfy patient's needs. So, Andrew's bridge seemed to be best treatment option in our case. Patient was informed about all these different treatment options and after the patient's approval, a fixed-removable prosthesis was planned for the prosthodontic rehabilitation of the partially edentulous condition in the maxillary and mandibular arch and for the closure of the bone defect. Patient agreed for the same.

The mobile lower lateral incisors $(32,42)$ were extracted and maxillary right central incisor (11) was endodontically treated. Diagnostic impressions were made using alginate impression material. Interocclusal record was taken. Diagnostics casts were then mounted on semiadjustable articulator using facebow record. Diagnostic wax-up was done. Maxillary left canine and first premolar and right central and lateral incisors and mandibular right and left canines, and first and second premolars (23, 24, 11, 12, 33, 43) were used as abutments, prepared, and final impressions made. The bar was fabricated in the wax pattern and casted along with copings. Esthetics, phonetics, and access for hygiene were evaluated during metal try-in (Fig. 4). Shade selection was performed. Ceramic firing was then done on the copings. After finishing and polishing, the crowns with the bar were cemented. With the crowns cemented in place, alginate impressions were made and stone cast was poured. The missing teeth were arranged in the wax rims and trial was done (Fig. 5). Acrylization

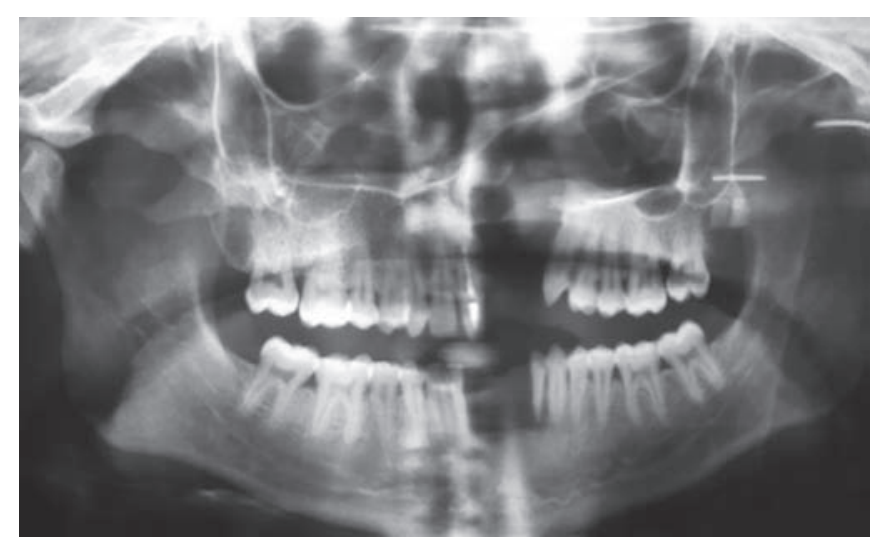

Fig. 3: Orthopantomogram 


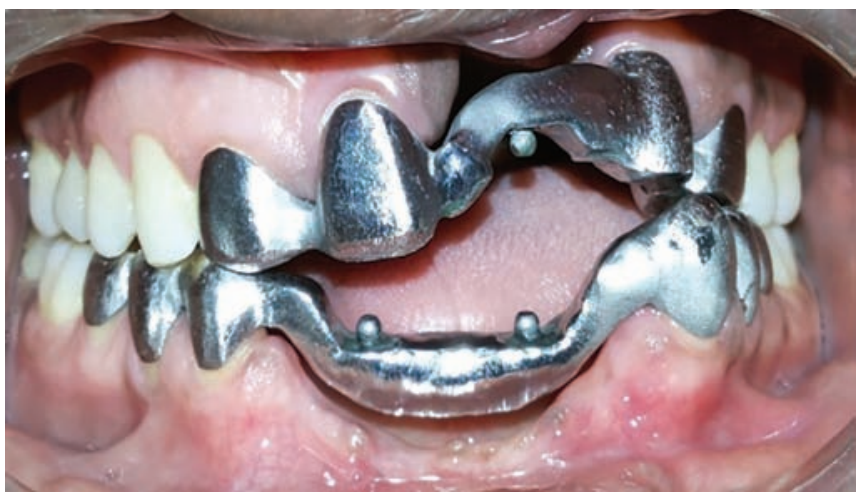

Fig. 4: Metal try-in

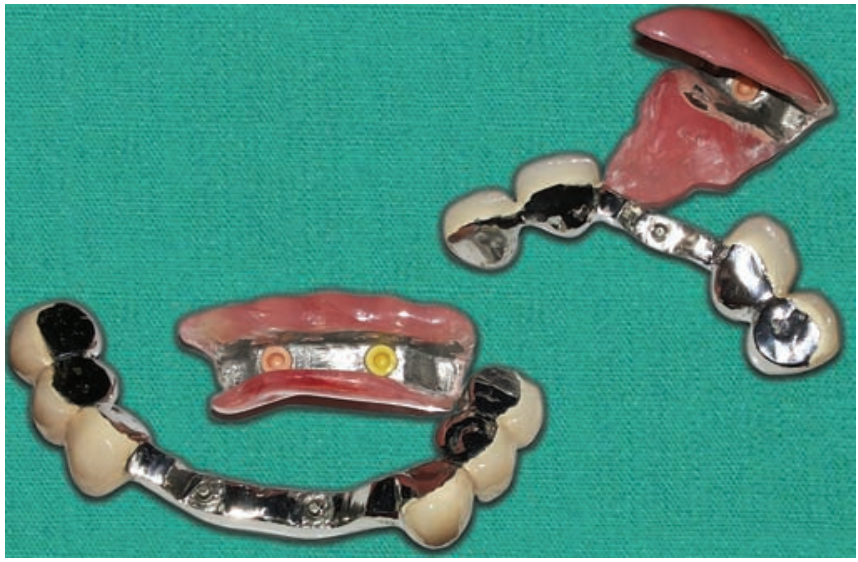

Fig. 6: Tissue surface of prosthesis

was done with the clip in the tissue surface of the denture base (Fig. 6). The removable segment was then attached over the bar attachment. Patient was trained to place and remove the removable components fabricated over the

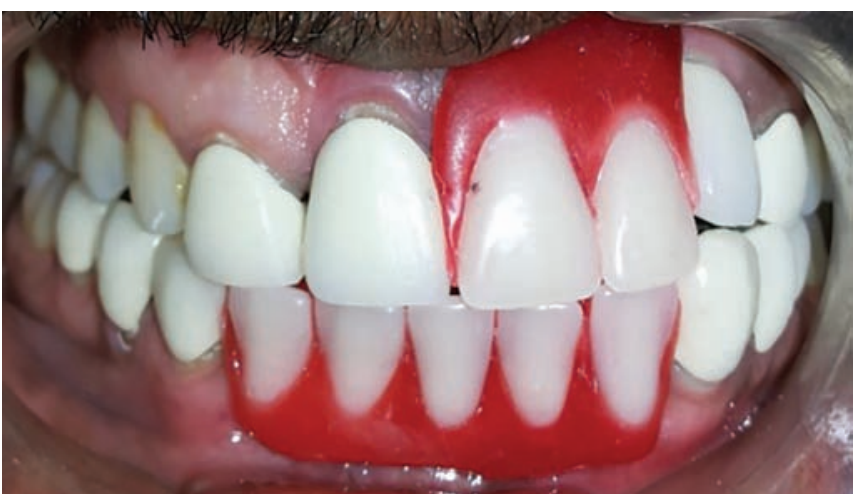

Fig. 5: Try-in

fixed components of Andrew's bridge (Figs 7A to D). Proper oral hygiene instructions were given. Periodic follow-up was done to evaluate patient's adaptability and satisfaction with the prosthesis (Fig. 8).

\section{DISCUSSION}

Localized alveolar ridge defect is an inevitable outcome in cases with trauma or congenital defects. Prosthodontic treatment of such large anterior ridge defects needs the restoration and rehabilitation of the soft and hard tissue defects, esthetics, and phonetics. This condition can pose a challenging situation to the dentist. "Localized alveolar ridge defect" refers to limited volumetric deficiency of hard and soft tissue within the alveolar process. ${ }^{2}$

Seibert has classified ridge defects into three classes. In class I ridge defect, horizontal or buccal tissue loss occurs with normal ridge height. In class II defect, vertical bone loss occurs with normal ridge width. In class III
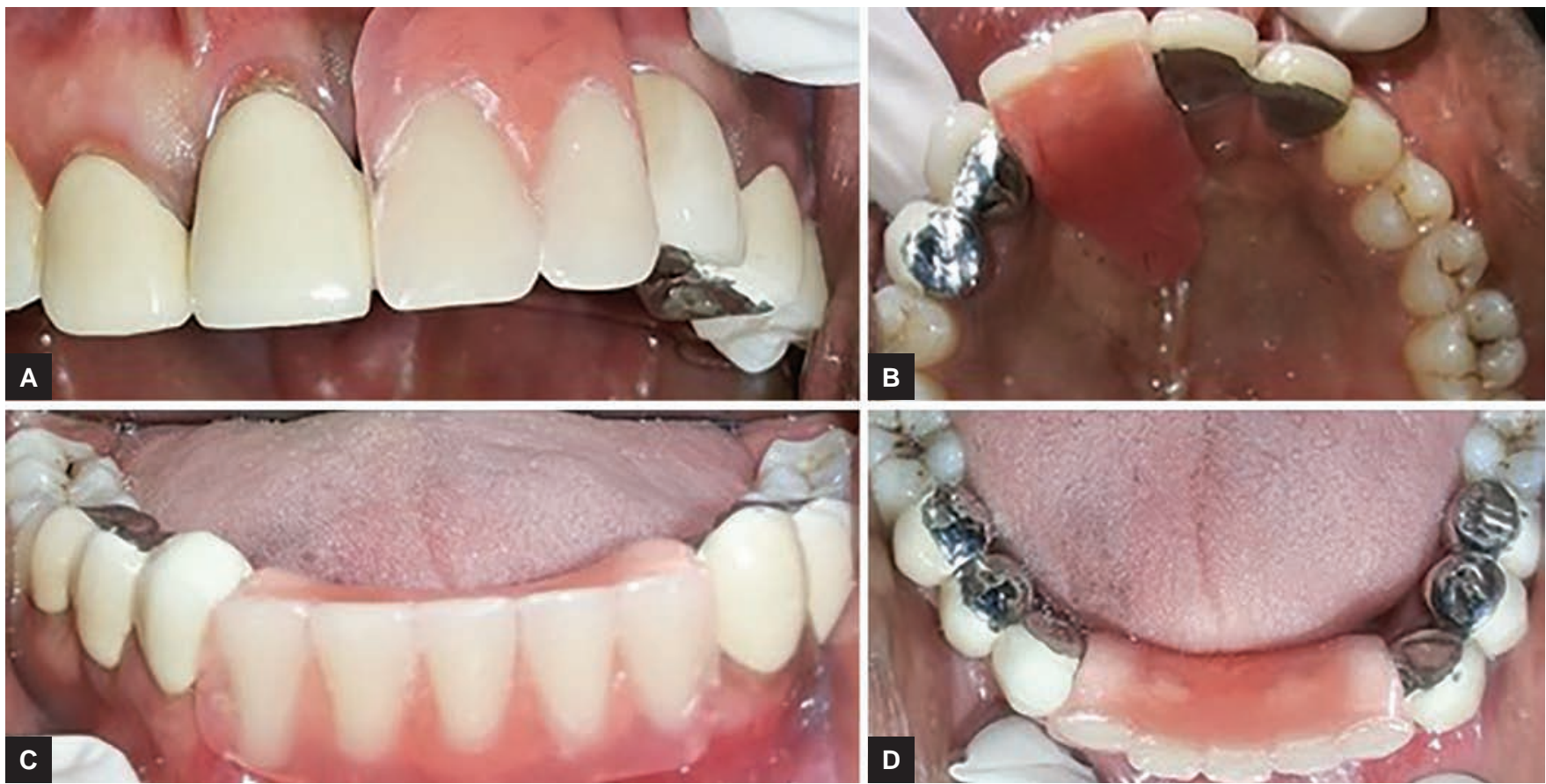

Figs 7A to D: Intraoral view after placement of prosthesis 


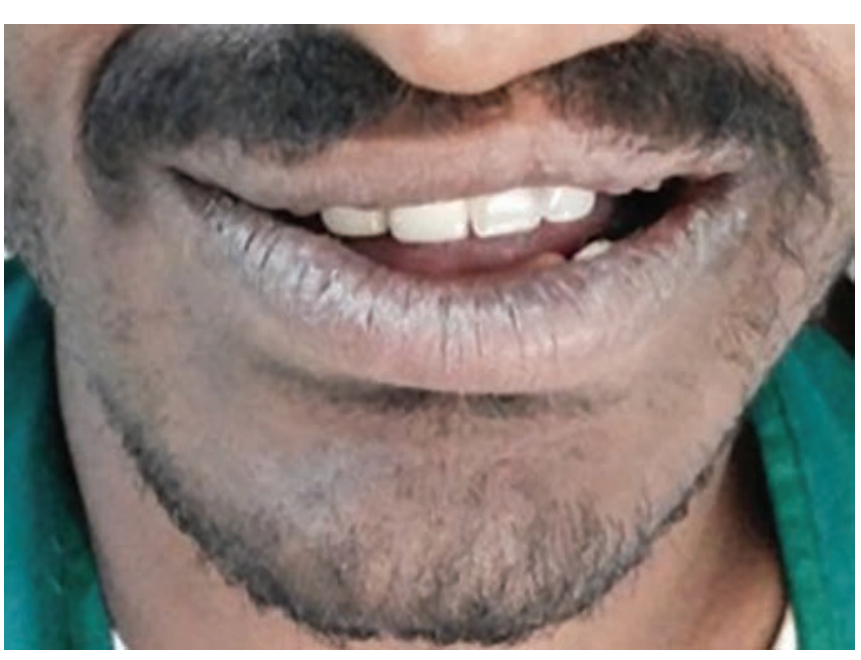

Fig. 8: Postoperative view

ridge defect, both horizontal and vertical bone losses occur. A semiquantitative analysis of severity of bone loss in vertical and horizontal directions was also postulated. Criteria for classification in the vertical and horizontal dimensions depend on the defect size in relation to the adjacent papillary tip. If the defect is less than $3 \mathrm{~mm}$, it is termed as mild defect; 2 to $6 \mathrm{~mm}$-moderate, and more than $6 \mathrm{~mm}$-severe. . $^{2,3}$

The vertical element of the defect is measured from the deepest point of the defect and an imaginary line running along the papillary tips of the adjacent teeth. The horizontal element is measured as the distance between the deepest point on the defect and an imaginary line running from the buccogingival margin and the cementoenamel junction of both the adjacent teeth. The classification helps in estimating the surgical and technical problems that can occur during the procedure. The vertical element of the ridge defect is more difficult to restore than the horizontal one. These defects may be restored by two methods: Preprosthetic surgery or restoration with fixed and/or removable prosthesis. ${ }^{3}$

Restoration of a localized alveolar ridge defect with a fixed prosthesis is incorporated with several esthetic complications like loss of papillae, formation of "black" interdental spaces, compromised phonetics, food accumulation under the pontic, and loss of buccal contour. ${ }^{4}$

The prosthetic treatment options for a short span edentulous clinical situation with severely deficient ridges include conventional FPD, implant-supported FPDs, RPD, or fixed-RPD. ${ }^{1}$ The FDP can restore function and esthetics only to a limited extent. The increased bone loss requires an increase in the length of the pontics that can lead to esthetic failure. Therefore, the defect will require correction by soft tissue grafts and bone grafts to render it suitable for FDP. Placement of implant requires preprosthetic surgeries, such as onlay grafts or alloplastic grafts, possibly with soft tissue regeneration that will require months of healing period, and the outcome may not be predictable. The conventional removable prosthesis does not satisfy patient's needs, and if not maintained properly, it causes decalcification and dental caries of the adjacent teeth, periodontal problems, such as inflammation of the gingival tissues, etc. ${ }^{1,5}$

Hence, when a proper diagnosis and treatment plan is made, Andrew's bridge provides a better therapeutic treatment. ${ }^{5}$ Replacement along with an acrylic denture flange for tissue defects is an added advantage as it does not require separate prosthesis for the gingival rehabilitation as in FDP. This is advantageous because it is not provided by the FDP, as it becomes more difficult to maintain. Andrew's bridge system is indicated in clinical conditions like extensive residual ridge defect, ridge defects due to trauma and/or surgical ablation, and cleft lip/palate patients with congenital or acquired bone defects. $^{6}$

The advantages of Andrew's bridge include better esthetics, hygiene maintenance, better adaptability, and phonetics. There is no palatal coverage and no soft tissue impingement on the surrounding structures, which comforts patient. The system acts as stress breaker while transmitting unwanted leverage forces. It is also an economical treatment option for the patient. ${ }^{7}$

It can be removed by the patient, thereby providing access for maintaining hygiene around the abutments and surrounding tissues. The pontic assembly can be relined as the ridge resorbs. It is more stable because it is completely tooth supported, and the occlusal forces are directed along the long axis of the abutment teeth. The flange of the pontic assembly can be contoured to improve esthetics and phonetics, and resist torque during mastication. Replacement of the teeth along with an acrylic denture flange is an added advantage as it does not require a separate prosthesis for the gingival defect as in the FDP. Andrew's bridge can be adapted to implant prosthesis very well. Since the prosthesis is attached to a bar retainer, the normal taste perception is maintained as the flange need not be extended palatally for support. Surgical correction of the defects using autografts / allografts followed by implant placement is an expensive treatment plan for some patients. Surgical procedures also require patient's consent and compliance. In conditions where conventional removable or fixed prosthesis is not a feasible option as in the case presented above, a third treatment option of Andrew's bridge can prove successful in restoring function, esthetics, speech, and closure of the defect. ${ }^{1,8}$

Very limited reports of the failure of such fixedremovable prosthesis are documented in the literature. The failures are mainly due to inadequate soldering. ${ }^{1}$ However, this was completely eliminated by attaching 
retainers to the bar in a single casting. The patient was comfortable with the final outcome and had pleasing esthetics and phonetics.

\section{CONCLUSION}

Replacing missing anterior teeth, especially, with a very large ridge defect is a challenging task to the clinicians. Such cases require restoration of the bone defect, esthetics, and phonetics along with replacement of the missing teeth. The conventional treatment options like FPD or a surgical option of implant placement may not justify the restoration of the ridge defects. In such conditions, a combination of fixed and removable restoration provides a good alternative. Andrew's bridge system is a fixedremovable prosthesis indicated in patients with large ridge defects. It successfully replaces the missing teeth along with complete closure of the defect and restores speech and esthetics. It also offers good retention and stability, thereby increasing patient comfort. We also achieved good adaptability, comfort, pleasing esthetics, and phonetics with a well-satisfied patient.

\section{REFERENCES}

1. Bhapkar P, Botre A, Menon P, Gubrellay P. Andrew's bridge system: an esthetic option. J Dent Allied Sci 2015 Oct;4(1):36-40.

2. Studer S, Naef R, Scharer P. Adjustment of localized alveolar ridge defects by soft tissue transplantation to improve mucogingival esthetics: a proposal for clinical classification and evaluation of procedures. Quintessence Int 1997 Dec;28(12):785-805.

3. Seibert JS, Cohen DW. Periodontal considerations in preparation for fixed and removable prosthodontics. Dent Clin North Am 1987 Jul;31(3):529-555.

4. Kaurani P, Kaur Samra R, Karunai M, Padiyar N. Prosthodontic rehabilitation of a case with an anterior ridge defect using Andrew's bridge. Indian J Dent Sci 2013 Jun;5(2):100-103.

5. Everhart R, Cavazos E Jr. Evaluation of fixed removable partial denture: Andrew's bridge system. J Prosthet Dent 1983 Aug;50(2):180-184.

6. Finley JM. Restoring the edentulous maxilla using an implant supported, matrix-assisted secondary casting. J Prosthodont 1998 Mar;7(1):35-39.

7. Shankar R, Raju AVR, Raju DS, Babu PJ, Kumar DRV, Rao B. A fixed removable partial denture treatment for severe ridge defect. Int J Dent Case Reports 2011 Aug;1(2):112-118.

8. DeBoer J. Edentulous implants: overdenture versus fixed. J Prosthet Dent 1993 Apr;69(4):386-390. 